Mehmet Akif Ersoy Üniversitesi Fen Bilimleri Enstitüsü Dergisi 10(1): $51-59$ (2019)

The Journal of Graduate School of Natural and Applied Sciences of Mehmet Akif Ersoy University 10(1): $51-59$ (2019)

Araştırma Makalesi / Research Paper

\title{
Klasik Su Verme-Temperleme Isıl İşlemine Göre Yenilikçi Su Verme- Ayrıştırma IsıI İşleminin Orta Karbonlu Çeliğin Mikroyapı ve Sertlik Özelliklerine Etkisi
}

\author{
Merve Şehnaz IŞYARLAR (iD 1*, Ferhat GÜL (iD) 1 \\ ${ }^{1}$ Gazi Üniversitesi, Teknoloji Fakültesi, Ankara \\ Geliş Tarihi (Received): 24.03.2019, Kabul Tarihi (Accepted): 13.05.2019 \\ $\square$ Sorumlu Yazar (Corresponding author*): nmisyarlar@hotmail.com \\ (C) +903122028801 且 +903122028947
}

ÖZ

Çeliklerin şekillendirilebilirliği bakımından süneklik, istenen bir malzeme özelliğidir. Sanayide sıkça kullanılan bir işlem olan klasik su verme-temperleme ısıl işlemi ile istenilen sünekliğin elde edilmesi mümkündür. Fakat sünekliğin artmasıyla çeliğin sertliğinin azalması istenmeyen bir etkendir. Yenilikçi su verme-ayrıştırma ısıl işlemi ile süneklik ve mukavemetin iyi bir kombinasyonunun elde edilmesi amaçlanmaktadır. Bu çalışmada 0,38 karbon miktarına sahip çeliğe uygulanan su verme-temperleme ve su verme-ayrıştırma ısıl işlemlerinin mikroyapı ve sertlik özelliklerinin karşılaştırılması yapılmıştır. Mikroyapıda varlığı gözlemlenen kalıntı östenite rağmen su verme-ayrıştırma ısıl işlemi ile elde edilen sertlik değerlerinin, klasik su verme-temperleme ısıl işlemine göre yüksek olduğu sonucuna varılmıştır.

Anahtar Kelimeler: Temperleme, Karbon ayrıştırma, Kalıntı östenit

\section{Effect of Innovative Quenching-Partitioning Heat Treatment on Microstructure and Hardness Properties of Medium Carbon Steel According to Traditional Quenching-Tempering Heat Treatment}

\begin{abstract}
Ductility in the formability of the steels is the desired material property. It is possible to obtain the desired ductility by the traditional quenching-tempering heat treatment which is often used in industry. However, decreasing the hardness of steel with increasing ductility is an undesirable factor. It is aimed to obtain a good combination of ductility and strength with the innovative quenching-partitioning heat treatment. In this study, microstructure and hardness properties of quenching-tempering and quenching-partitioning heat treatments applied to steel having 0.38 carbon content were compared. Despite the retained austenite observed in the microstructure, it was concluded that the hardness values obtained by the quenching-partitioning heat treatment were higher than the traditional quenching-tempering heat treatment.
\end{abstract}

Keywords: Tempering, Carbon partitioning, Retained austenite 


\section{Giriş}

Otomotiv ve diğer endüstriler tarafından yüksek süneklik ve şekil verilebilirlik ile yüksek mukavemet özelliklerinin birleşimi aynı zamanda daha düşük maliyetle üretilmesi gibi sebeplerden dolayı kullanım talebinin artması, 3 . Nesil Geliştirilmiş Yüksek Dayanımlı Çeliklerin geliştirilmesi için çalışmaların yoğunlaşmasına neden olmuştur (Wang ve ark., 2011; Qu, 2013; Kolk, 2014; Tariq ve Baloch, 2014; De Diago-Calderón ve ark., 2015). Bu nedenle, daha iyi mekanik özellikler için faz dönüşümü yoluyla mikroyapının geliştirilmesi üzerinde durulmuştur (Wang ve ark., 2011). Bu tip çelik üretmenin olası yollarından biri, Su verme ve Ayrıştırma (Quenching and Partitioning, Q-P) adlı yenilikçi bir tavlama ısı işlemidir (Arlazarov ve ark., 2015). Q-P işlemi, 2003 yılında Speer ve arkadaşları tarafından ortaya konulmuştur (Gao ve ark, 2013; Arlazarov ve ark., 2015). Bu ısıl işlemin amacı, yüksek dayanımlı çeliklerin mekanik özelliklerini geliştirmek için mikroyapıda martenzitik matris ve karbonca zengin kalıntı östenit elde etmektir (Liu ve ark., 2011; Wang ve ark., 2016).

Q-P işlemi su verme ve ayrıştırma olarak iki adımdan oluşur. İlk olarak çelik tamamen östenit mikroyapı veya kritik sıcaklıklar arası (Ac1-Ac3) mikroyapıyı $(\alpha+\gamma)$ elde etmek için tavlanır. Ardından çelik, martenzit başlama (Ms) ve martenzit bitiş (Mf) sıcaklıkları arasında ki bir sıcaklığa su verilerek mikroyapıda martenzit ve dönüşmemiş östenit meydana gelmesi sağlanır. Ayrıştırma adımı için çelik ya su verildiği sıcaklıkta izotermal olarak ya da su verildiği sıcaklığın üzerinde ki bir sıcaklıkta ayrıştırma süresi olarak belirlenen sürede o sıcaklıkta tutulur. Daha sonra çeliğe oda sıcaklığında su verilmesiyle ısıl işlem tamamlanmış olur (Bagliani ve ark., 2013; Hao ve ark., 2016; HajyAkbary ve ark., 2016). Ayrıştırma aşamasında, karbon aşırı doymuş martenzitten komşu dönüşmemiş östenit içerisine yayınır ve oda sıcaklığına su verme adımı sırasında karbon kazanmış halde ki östenitin yeni martenzitlere dönüşmeden oda sıcaklığında kararlı halde kalması sağlanır (Gao ve ark., 2013; Wang ve Speer, 2013; De Diago-Calderón ve ark., 2015). Bu yöntemle, mikroyapıda karbon kaybetmiş martenzit ve karbon kazanmış östenitin varlığı, dayanım ile sünekliğin bir arada oluşmasını sağlamak için elde edilir (Gao ve ark., 2013; Speer ve ark., 2015).

Su verme ve ayrıştırma ısıl işlemi, mekanik özellikleri esas olarak temperlenmiş martenzit mikroyapısına bağıı olan geleneksel su verme-tempereleme (Quenching and Tempering, Q-T ) ısıl işleminden oldukça farklıdır (Wang ve ark., 2011). Q-P ısıl işlemi uygulanan çelikler, geleneksel Q-T çeliklerine kıyasla önemli miktarda kalıntı östenit miktarına sahiptirler (Hao ve ark., 2016).
Q-P ısıl işleminde, ayrıştırma adımı sırasında dönüşmemiş östenitlerin tamamının oda sıcaklığında kararlı halde kalması için karbonca zenginleşmesinin yetersiz kalması halinde, oda sıcaklığına su verme süresince dönüşmemiş östenitlerin bir kısmının yeni martenzitlere dönüşmesi kaçınılmazdır. Bu yeni oluşan martenzitler yüksek karbon içeriğine sahiptir ve çeliğin sünekliği için zararlıdır (HajyAkbary ve ark., 2016).

Ayrıştırma adımı sırasında martenzitten östenite yayınması istenen karbonu tüketme eğilimde olan östenitin ferrit ve sementite ayrışması (beynit ve perlit oluşumu) ve karbür (geçiş karbürleri) çökelmesi gibi rekabetçi reaksiyonları engellemek için uygun alaşımlama yapılmalıdır (Edmonds ve ark., 2006; De Diago-Calderón, 2015).

Karbonca zengin yarı kararlı kalıntı östenitin yararlı özelliklere sahip olduğu düşünülmektedir. Çünkü kalıntı östenit, deformasyon sırasında Dönüşüm Etkili Plastisite (Transformation Induced Plasticity, TRIP) etki göstermesiyle önemli ölçüde malzemenin enerji emme ve şekil verilebilirliğini destekleyici özelliğe sahiptir (Santofimia ve ark., 2009).

Kalıntı östenitin kararlılığının olabildiğince fazla karbon içeriğiyle sağlamak için çelik içerisine silisyum, alüminyum, fosfor, nikel gibi karbür oluşumunu engelleyen elementler eklenir (Yang ve ark., 2011; Hauserova ve ark., 2011; Karam-Abian ve ark., 2016). Çelik içerisine silisyum ilavesi sementit oluşumunu geciktirebilir, hatta bunu engelleyebilir. Alüminyum ve fosfor elementlerinin de temperleme reaksiyonlarını geciktirmede silisyum ile benzer özellikler gösterir. Bu gibi elementler, Q-P ısıl işleminde kritik rol oynamaktadır (De Diago-Calderón, 2015). Ayrıca karbür oluşumuna neden olan niyobyum, molibden ve vanadyum gibi elementlerin çelik içerisine eklenmesinden kaçınılmalıdır (Wang ve ark., 2010; Zhou ve ark., 2011).

Bu çalışmada orta-karbonlu çelik malzemeye uygulanan klasik su verme-temperleme ısıl işlemi ile yenilikçi su verme-ayrıştırma ısıl işleminin, sertlik ve mikroyapı özellikleri karşılaştırılmıştır. Su verme-ayrıştırma ısıl işlemi ile elde edilen mikroyapının, klasik su verme-temperleme ısıl işlemine göre sahip olduğu farklııklar Taramalı Elektron Mikroskobu (Scanning Electron MicroscopySEM) mikroyapı görüntüleri ile incelenmiştir. Klasik su verme-temperleme ısıl işlemi ile elde edilen temperlenmiş martenzit mikroyapısından farklı olarak su vermeayrıştırma ısıl işlemiyle, mikroyapıda sahip olunan kalıntı östenitin varlığına rağmen su verme-temperleme ısıl işlemine göre sertlik değerlerine ilişkin bulgular grafikler halinde verilmiştir. 


\section{MATERYAL VE YÖNTEM}

Isıl işlemlerde kullanılan çeliğin kimyasal bileşimi Tablo 1 ' de, SEM ve optik mikroskop mikroyapı görüntüleri Şekil 1' de gösterilmektedir. Bu çelikten, Isıl işlem deneylerinde kullanılmak üzere $40 \mathrm{~mm}$ çapında ve $10 \mathrm{~mm}$ boyunda 5 tane numune hazırlanmıştır.
Tablo 1. Isıl işlemlerde kullanılan çeliğin kimyasal bileşimi (ağırlıkça \%)

\begin{tabular}{|c|c|c|c|c|c|}
\hline $\mathbf{C}$ & $\mathbf{S i}$ & $\mathbf{M n}$ & $\mathbf{C r}$ & $\mathbf{M o}$ & $\mathbf{N i}$ \\
\hline 0.38 & 0.34 & 0.70 & 0.76 & 0.19 & 1.52 \\
\hline
\end{tabular}

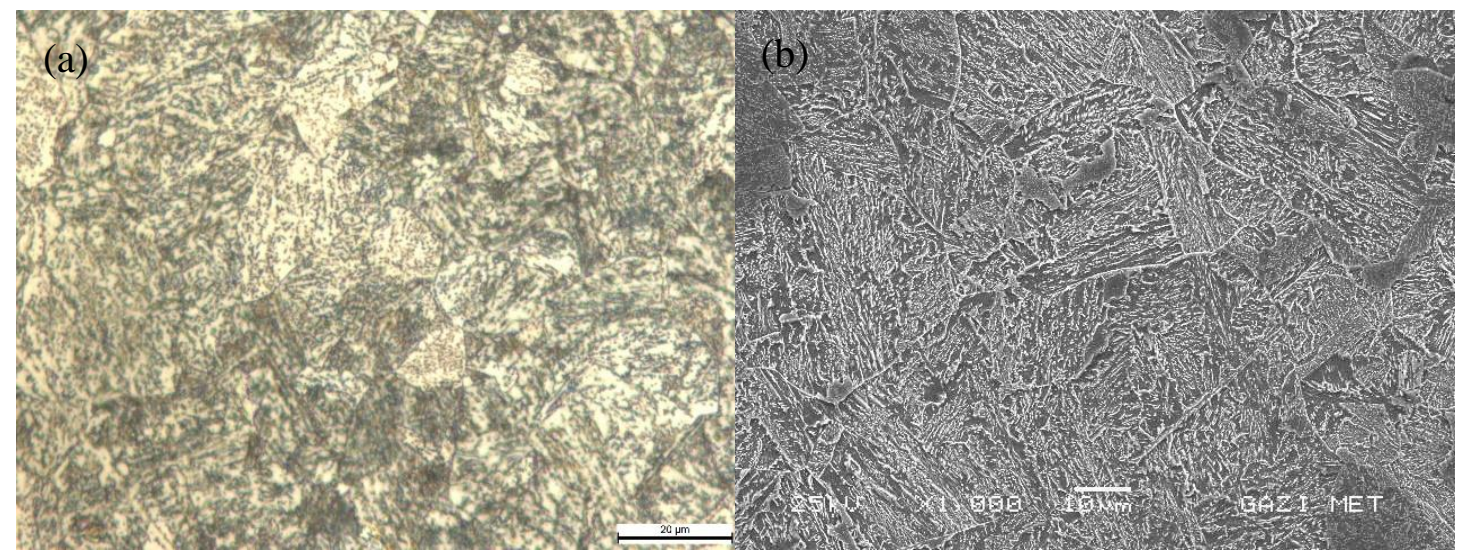

Şekil 1. Isıl işlemler öncesi orta karbonlu çeliğin perlitik mikroyapısı: (a) optik mikroskop ve (b) SEM görüntüleri

Bu numunelerden bir tanesine, Şekil 2' de gösterildiği gibi klasik su verme-temperleme ısıl işlemi (QT numunesi) ve diğer 4 numuneye Şekil 3' te gösterildiği gibi farklı sıcaklık ve sürelerde su verme-ayrıştırma ısıl işlemleri uygulanmıştır. Su verme-temperleme ısıl işlemi için QT numunesi, $885^{\circ} \mathrm{C}$ ' de 30 dakika östenitlendikten sonra oda sıcaklığında suda su verilmiştir. Daha sonra temperleme işlemi için $555^{\circ} \mathrm{C}$ ' de fırında 1 saat bekletilip havada soğumaya bırakıımıştır.

Deneylerde kullanılan çeliğin martenzit başlangıç sıcakıı̆ı $\left(M_{s}\right)$ aşağıda verilen formüle göre hesaplanmıştır (Denklem 1) (Chipalkatti, 1999).

$$
\begin{aligned}
\mathrm{M}_{\mathrm{S}}\left({ }^{\circ} \mathrm{C}\right)= & 539-423(\mathrm{~A} \breve{\mathrm{g}} \% \mathrm{C})-30.4(\mathrm{~A} \breve{\mathrm{g}} \% \mathrm{Mn})- \\
& 17.7(\mathrm{Ag} \% \mathrm{Ni})-12.1(\mathrm{~A} \breve{\mathrm{g}} \% \mathrm{Cr})-7.5(\mathrm{~A} \breve{\mathrm{g}} \% \mathrm{Mo})- \\
& \text { 7.5( } \mathrm{A} \mathrm{g} \% \mathrm{Si})
\end{aligned}
$$

Bu formül hesabına göre su verme-ayrıştırma ısıl işlemi uygulanan çeliğin martenzit başlangıç sıcaklığı $317^{\circ} \mathrm{C}$ olarak kullanılmıştır.

Su verme-ayrıştırma işlemi için tüm numuneler $885^{\circ} \mathrm{C}$ ' de 30 dakika östenitleme işlemine tabi tutulduktan sonra
QP1 ve QP2 numuneleri $250^{\circ} \mathrm{C}$ ' ye, QP3 ve QP4 numuneleri ise $280^{\circ} \mathrm{C}$ ' ye su verilmiştir. Tüm Q-P numuneleri ilk su verme adımında $120 \mathrm{~s}$ tutulmuştur. Daha sonra ayrıştırma adımı için tüm Q-P numuneleri $350^{\circ} \mathrm{C}$ ' ye ısıtılıp hepsi için sırasıyla $10 \mathrm{~s}$ ve $100 \mathrm{~s}$ bekletilmiştir. Tablo 2' de su verme-ayrıştırma ısı işlemi uygulanmış numunelerin işlem değişkenleri verilmektedir. Isıl işlemlerde östenitleme için kutu tipi tav fırını, ısıtma ve soğutma işlemleri için içerisinde $\% 50$ sodyum nitrat, $\% 50$ potasyum nitrat karışımından oluşan iki adet tuz banyosu kullanılmıştır.

Tablo 2. Su verme-ayrıştırma ısıl işlem değişkenleri ( $T_{Q}$ ve Tp sırasıyla ilk su verme sıcaklığını ve ayrıştırma sıcaklığını temsil etmektedir)

\begin{tabular}{|l|c|c|c|}
\hline & $\mathbf{T}_{\mathbf{Q}}\left({ }^{\circ} \mathbf{C}\right)$ & $\mathbf{T}_{\mathbf{P}}\left({ }^{\circ} \mathbf{C}\right)$ & $\begin{array}{c}\text { Ayrıştırma } \\
\text { süresi(s) }\end{array}$ \\
\hline QP1 & 250 & 350 & 10 \\
\hline QP2 & 250 & 350 & 100 \\
\hline QP3 & 280 & 350 & 10 \\
\hline QP4 & 280 & 350 & 100 \\
\hline
\end{tabular}




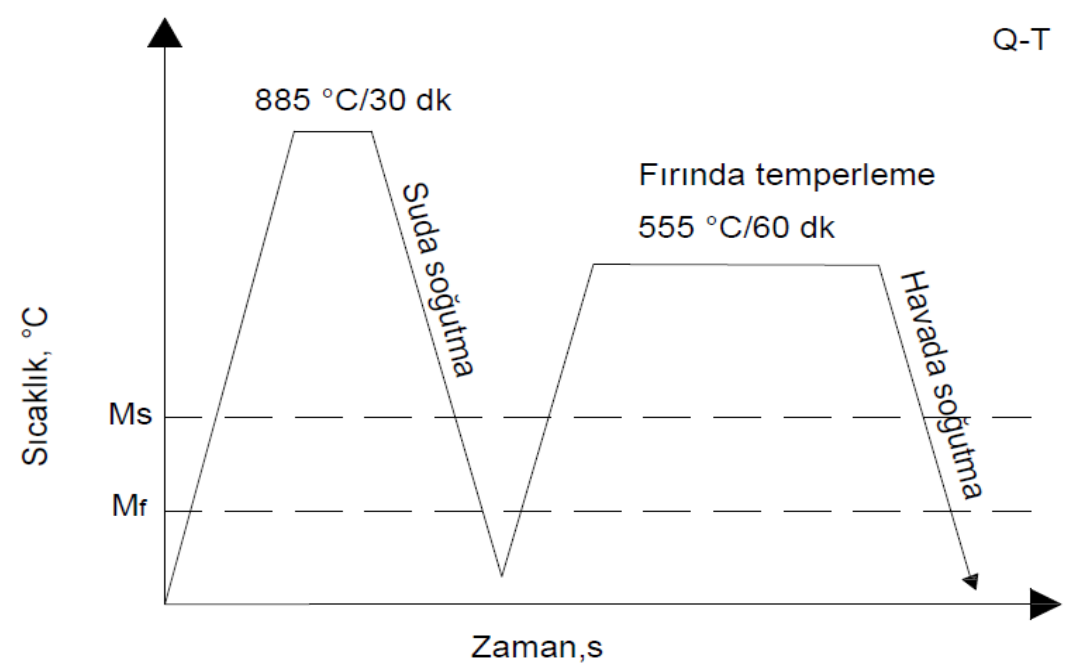

Şekil 2. Klasik su verme-temperleme ısıl işlemi sıcaklık-zaman grafiği

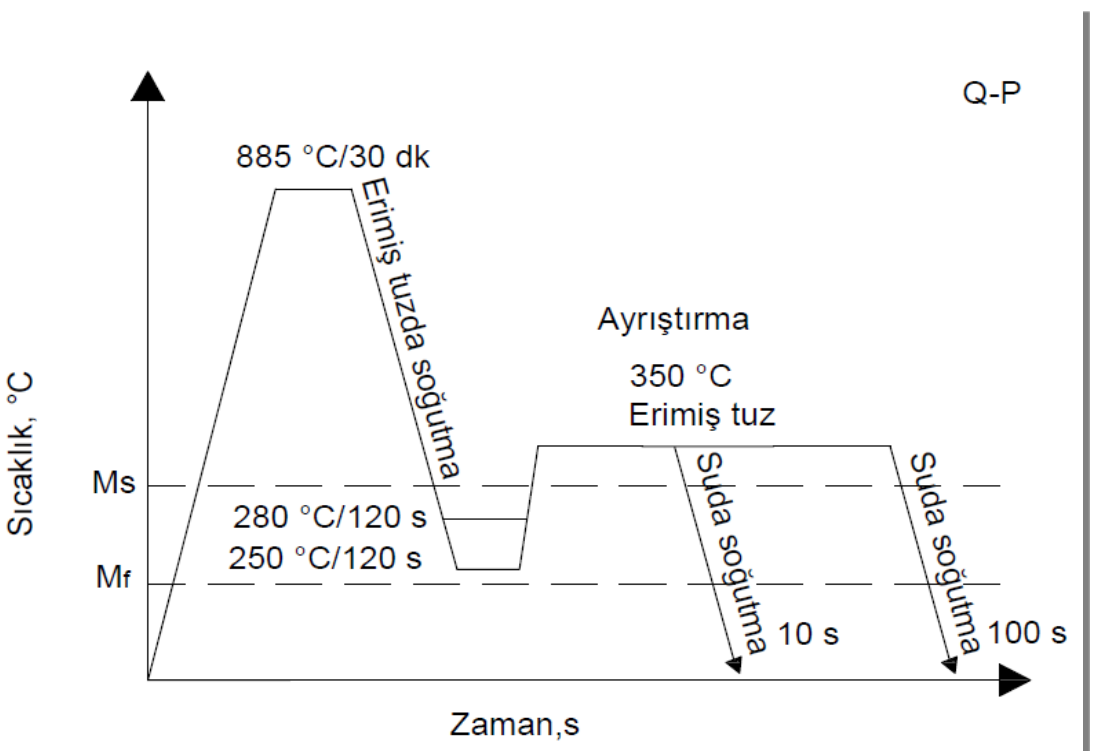

Şekil 3. Su verme-ayrıştırma ısıl işlem sıcaklık-zaman grafiği

Tüm numuneler, zımparalama ve parlatma yüzey işlemlerinden sonra \%2' lik Nital ile dağlanarak metalografik inceleme için hazır hale getirilmiştir. Numunelerin mikroyapı görüntüleri, Jeol JEM 6060LV Tarama Elektron Mikroskobu (SEM) kullanılarak elde edilmiştir. Numunelerin sertlik ölçümleri, $2.5 \mathrm{~mm}$ karbür bilya ile $187.5 \mathrm{kgf}$ yük kullanılarak Brinell Sertlik yöntemiyle yapılmıştır.

Illk su verme sıcaklığındaki martenzit miktarı KoistinenMarburger eşitliği ile tahmin edilmiştir (Denklem 2) (Wang ve ark., 2010; Tan ve ark., 2014).

$\mathrm{f}_{\mathrm{i}}^{\mathrm{M}}=1-\exp \left[-1.1 \times 10^{-2} \times\left(\mathrm{M}_{\mathrm{s}}-\mathrm{T}_{\mathrm{Q}}\right)\right]$
Eşitlikte $\mathrm{f}_{\mathrm{i}}^{\mathrm{M}}$ martenzitin hacim oranını, TQ ise ilk su verme sıcaklığını temsil etmektedir. Ms, deneyde kullanılan çelik için martenzit başlama sıcaklığını ifade etmektedir.

\section{BULGULAR VE TARTIŞMA}

Orta karbonlu çeliğe uygulanan klasik su verme-temperleme ısıl işlemi sonucunda sahip olduğu mikroyapı optik mikroskop görüntüsü Şekil 4' te ve SEM mikroyapı görüntüleri farklı büyütmelerde Şekil 5' te gösterilmektedir. Su verme-temperleme ısıl işleminden sonra mikroyapı, temperlenmiş martenzit yapıdadır. Şekil 5 ' te gösterildiği gibi martenzit bloklar içerisinde dağılmış sementit taneleri gözlenmektedir. Su verilerek martenzit yapıya sahip 
olan çeliklerin, temperleme işlemiyle sertliğinde azalma olurken tokluğunda artış gerçekleşmektedir (Digges ve ark., 1966).

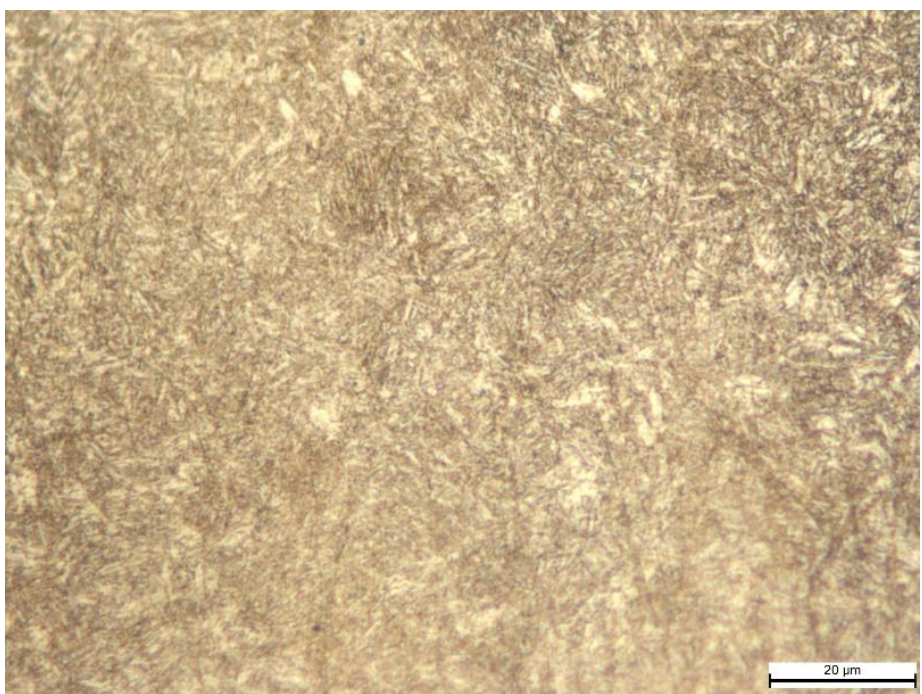

Şekil 4. Klasik su verme-temperleme ısıl işlemi uygulanan çeliğin optik mikroskop görüntüsü

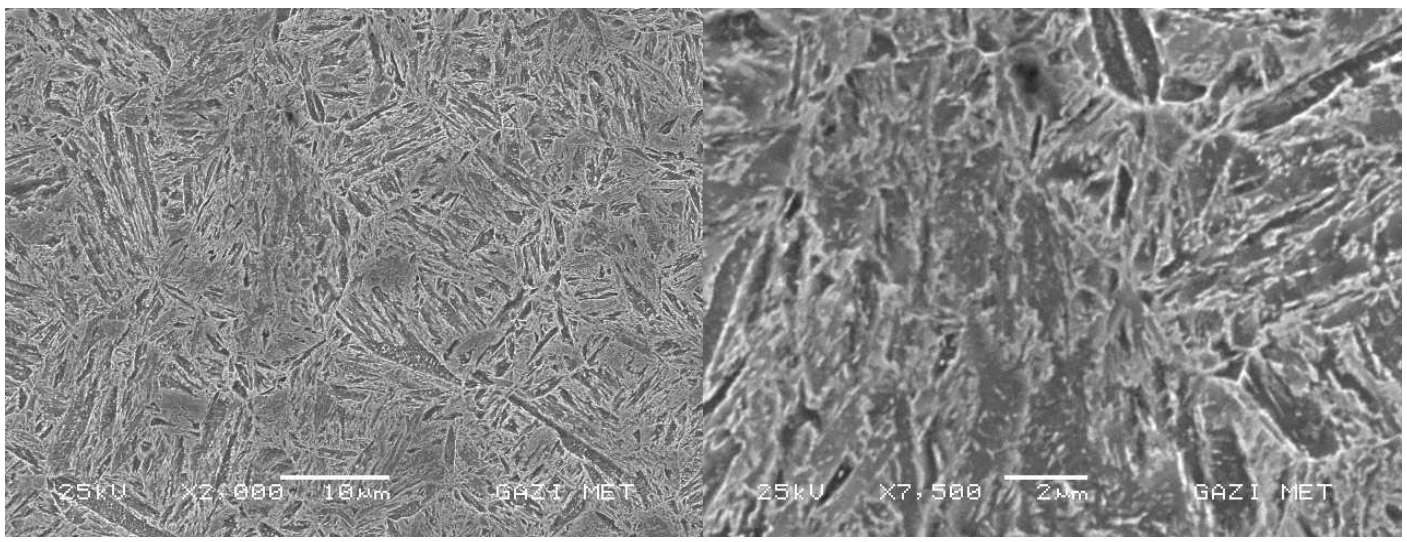

Şekil 5. Klasik su verme-temperleme ısıl işlemi uygulanan çeliğin SEM mikro fotoğrafları

Şekil 6' da su verme-ayrıştırma ısıl işlemi uygulanan numunelerin mikroyapı görüntüleri gösterilmektedir. Mikroyapılar, iğnemsi martenzit $(\mathrm{ML})$, plaka martenzit $(\mathrm{MP})$ ve martenzit bloklarının sınırlarında film şeklinde kalıntı östenitlerden (RA) oluşmaktadır. İlk su verme adımında oluşan martenzitler, ayrıştırma adımında karbon kaybederek temperlenmiş martenzitlere $\left(\mathrm{M}_{1}\right)$ ve ayrıştırma adımında ise yeterli karbon alımı gerçekleşemeyen dönüşmemiş östenitler, ikinci su verme adımında karbon oranı yüksek blok şeklinde yeni martenzitlere $\left(\mathrm{M}_{2}\right)$ dönüşmektedir (Bagliani ve ark., 2013; HajyAkbary ve ark., 2016). 


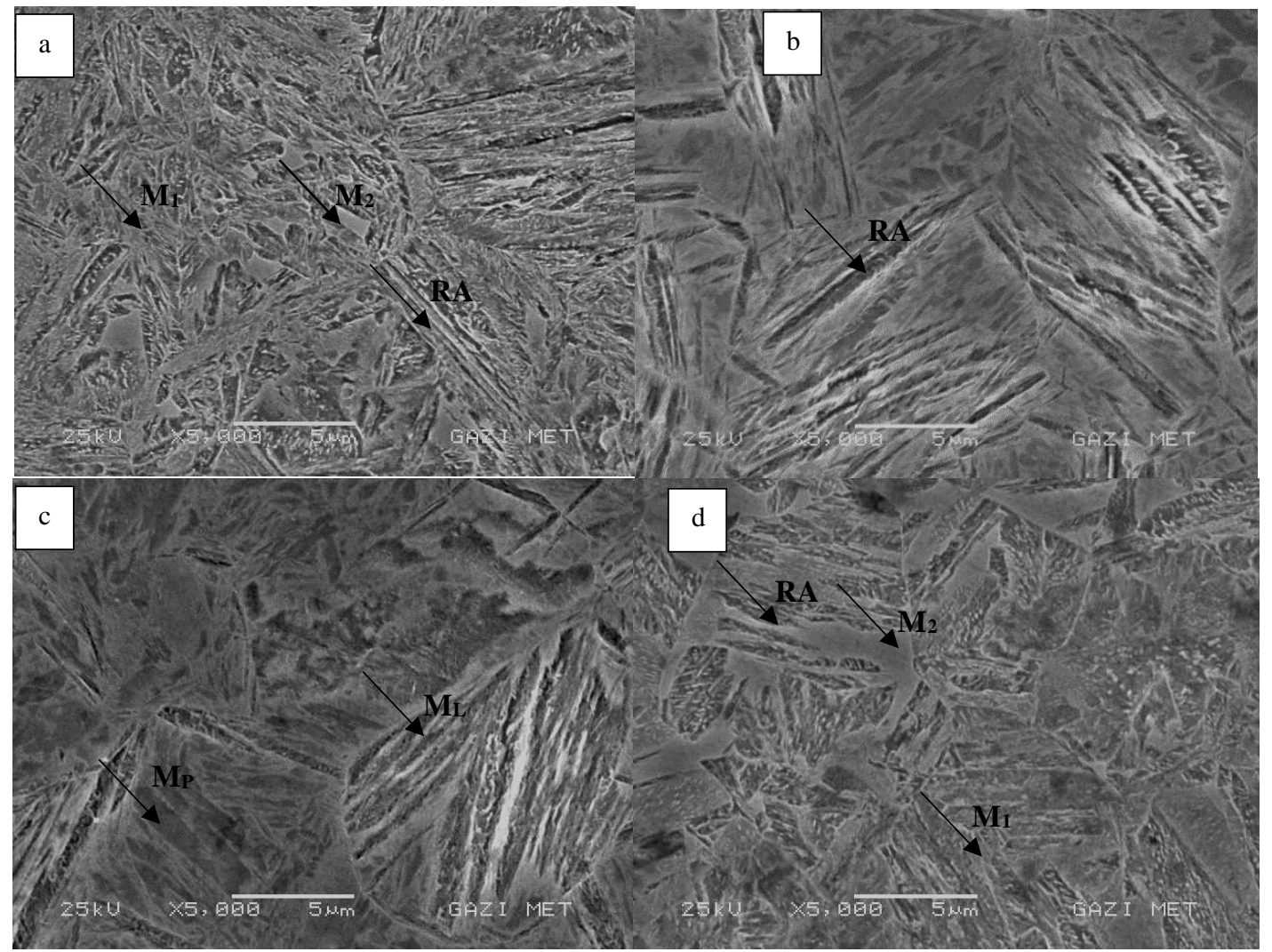

Şekil 6. Su verme-ayrıştırma ısıl işlemi uygulanan çeliklerin SEM görüntüleri: (a) QP1 (250/350 $\left.{ }^{\circ} \mathrm{C}-10 \mathrm{~s}\right)$, (b) QP2 $\left(250 / 350{ }^{\circ} \mathrm{C}-100 \mathrm{~s}\right),(\mathrm{c}) \mathrm{QP} 3\left(280 / 350^{\circ} \mathrm{C}-10 \mathrm{~s}\right)$ ve (d) QP4 $\left(280 / 350^{\circ} \mathrm{C}-100 \mathrm{~s}\right)$

Su verme-temperleme ısıl işlemi sonucunda elde edilen mikroyapı ile su verme-ayrıştırma sonucunda elde edilen mikroyapılar birbirlerinden farklı olduğu Şekil 5' te ve 6 ' da açıkça görülmektedir. Su verme- ayrıştırma ısıl işlemi sonucunda farklı yapılarda martenzit fazı ve kalıntı östenitten oluşan karmaşık mikroyapı, çeliğin sahip olduğu mekanik özellikleri etkilemektedir (Liu ve ark., 2011). Mikroyapıda ince film şeklinde bulunan kalıntı östenit fazı çeliğin sahip olması istenen TRIP etki için yararlıdır. Bunun nedeni film şeklindeki östenitlerin daha fazla yüzey alanına sahip olmasıdır ve bu, izotermal ayrıştırma adımında daha fazla karbon toplayarak ikinci su verme adımı sırasında yeni martenzitlere dönüşmeden kararlı halde kalmasını sağlar (Dong ve ark., 2018). Blok tipi östenitler, ayrıştırma adımı sırasında yeterli karbonu toplayamadıklarından ikinci su verme adımında yeni martenzitlere $\left(\mathrm{M}_{2}\right)$ dönüşmektedir (Karam-Abian ve ark., 2016).

Şekil 7' de bütün numunelerin sertlik değerleri gösterilmektedir. Sertliğin, su verme-ayrıştırma ısıl işlemiyle su verme-temperleme ısıl işlemine göre artıı̆ı görülmektedir. 
Klasik Su Verme-Temperleme IsıI İşlemine Göre Yenilikçi Su Verme-Ayrıştırma Isıl İşleminin Orta Karbonlu Çeliğin Mikroyapı ve Sertlik Özelliklerine Etkisi

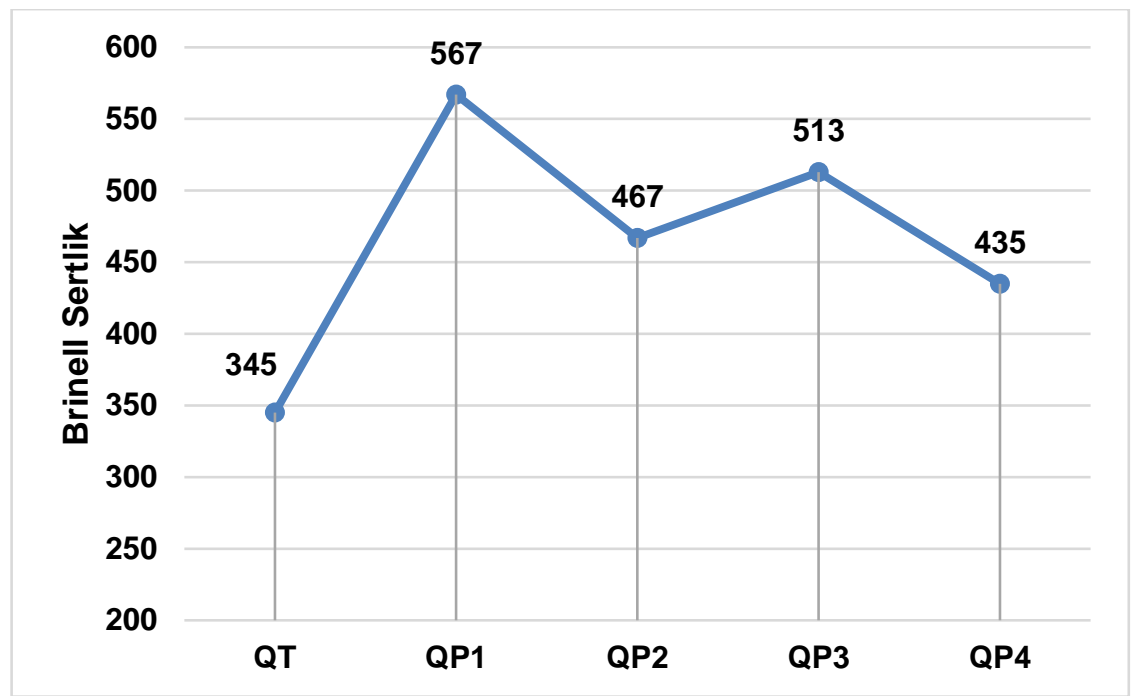

Şekil 7. Su verme-temperleme ve su verme-ayrıştırma ısıl işlemi uygulanan numunelerin sertlik değerleri

Su verme-ayrıştırma ısıl işlemi uygulanan çeliklerin, ısıl işlem değişkenlerine göre değişen sertlik değerleri Şekil 8' de gösterilmektedir. İlk su verme sıcaklığının artmasıyla ve artan ayrıştırma süresiyle sertlik değerlerinde azalma gözlemlenmektedir. İlk su verme sıcaklığının $250^{\circ} \mathrm{C}$ ' den $280^{\circ} \mathrm{C}$ ' ye yükseltilmesi, ayrıştırma adımından önce bu su verme sıcaklıklarında dönüşmemiş östenit miktarlarının sırasıyla, \%36 ve \%50 olduğu denklem 1 ile hesaplanmış ve oda sıcaklığında elde edilecek dönüşmemiş östenit miktarının artmasına neden olduğu sonucuna varılmıştır. Ayrıştırma süresinin artmasıyla ilk su verme adımında oluşan martenzitlerden dönüşmemiş östenitlere karbon difüzyonu için gerekli süre artmakta ve bu süre içerisinde karbon kaybeden martenzitlerin sahip olduğu gerinim enerjisi azalmaktadır. Martenzitlerin karbon kaybederek kademeli olarak temperlenmiş martenzite dönüşmesi çeliğin sertliğinin azalmasına neden olmaktadır (Krauss, 1999; Karam-Abian ve ark., 2016).

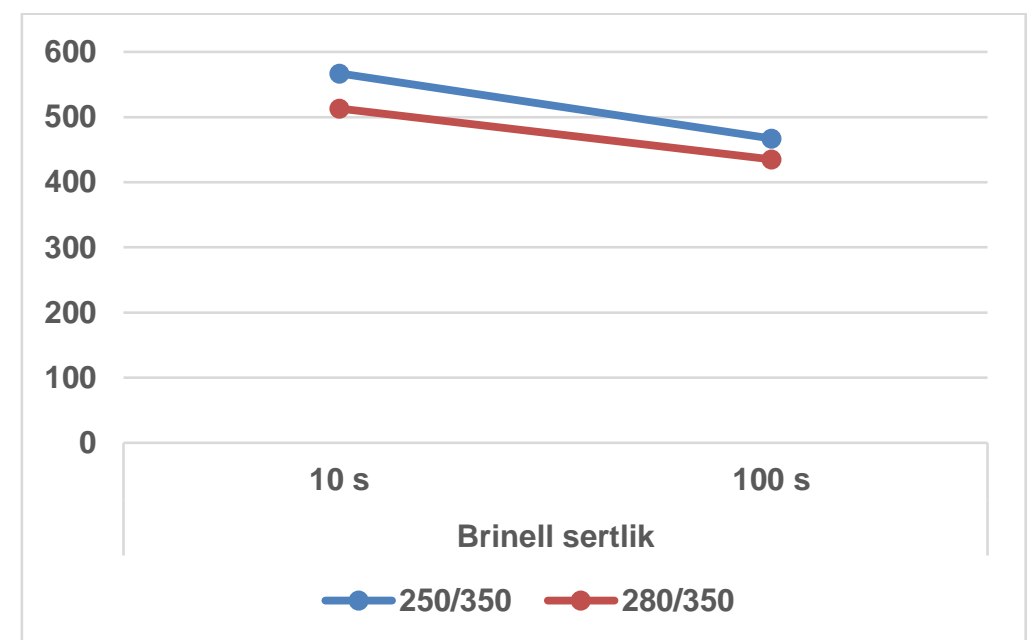

Şekil 8. Su verme-ayrıştırma ısıl işlemi uygulanan numunelerin ayrıştırma süresine göre değişen sertlik değerleri

\section{SONUÇLAR}

Bu çalışmadan elde edilen sonuçlar aşağıda maddeler halinde aşağıda verilmiştir;

- Karbon oranı \%0.38 olan alaşımlı çeliğin, $550{ }^{\circ} \mathrm{C}$ ' de 1 saat temperlenmesiyle temperlenmiş martenzit mikroyapısı elde edilmiştir.
- Su verme-ayrıştırma ısıl işlemiyle elde edilen çeliğin mikroyapısı, kalıntı östenitten, temperlenmiş martenzitten ve blok şeklinde yeni martenzitlerden oluşmaktadır.

- QP numunelerinin QT numunesine göre sahip oldukları yüksek sertlik değerleri, mikroyapıda çoğunlukta bulunan martenzit fazının varlığıyla açıklanmaktadır. 
- QP numunelerinin SEM görüntülerinde, mikroyapıda martenzit levhaları sınırlarında ince film halinde kalıntı östenitlerin bulunduğu gözlemlenmiştir.

- Su verme-ayrıştırma ısıl işleminde ilk su verme sıcaklığının arttırılması $\left(250^{\circ} \mathrm{C}\right.$ ' den $280^{\circ} \mathrm{C}$ ' ye), izotermal ayrıştırma adımından önce elde edilen dönüşmemiş östenit miktarını arttırmaktadır. Buna göre ayrıştırma adımından sonra oda sıcaklığında elde edilecek kaIıntı östenit miktarının daha yüksek olacağı tahmin edilmektedir.

- Su verme-ayrıştırma ısıl işleminde ayrıştırma süresinin arttırılmasıyla (10s' den 100s' ye), sertlik değerleri düşmektedir.

Klasik su verme-temperleme ısıl işleminden farklı olarak su verme-ayrıştırma ısıl işlemi ile elde edilen yüksek sertlik değerlerinin yanında mikroyapıda bulunan kalıntı östenitlerin, çeliğin sünekliğine katkı sağlayacağı düşünülmektedir.

\section{KAYNAKLAR}

Arlazarov, A., Bouaziz, O., Masse, J. P., Kegel, F. (2015). Characterization and modeling of mechanical behavior of quenching and partitioning steels. Materials Science and Engineering: A 620: 293-300.

Bagliani, E. P., Santofimia, M. J., Zhao, L., Sietsma, J., Anelli, E. (2013). Microstructure, tensile and toughness properties after quenching and partitioning treatments of a mediumcarbon steel. Materials Science and Engineering: A 559: 486-495.

Chipalkatti, J. (1999). Modeling of Austenite Decomposition in an AISI 4140 Steel. Doctoral Dissertation, University of British Columbia.

De Diego Calderón, M. I. (2015). Mechanical Properties of Advanced High-Strength Steels Produced via Quenching And Partitioning. Doctoral Dissertation, Universidad Carlos III de Madrid.

De Diego-Calderón, I., De Knijf, D., Monclús, M. A., MolinaAldareguia, J. M., Sabirov, I., Föjer, C., Petrov, R. H. (2015). Global and local deformation behavior and mechanical properties of individual phases in a quenched and partitioned steel. Materials Science and Engineering: A 630: 27-35.

Digges, T. G., Rosenberg, S. J., Geil, G. W. (1966). Heat Treatment and Properties of Iron and Steel. Natıonal Bureau of Standards Gaithersburg Md.

Dong, H. Y., Wu, K. M., Wang, X. L., Hou, T. P., Yan, R. (2018). A comparative study on the three-body abrasive wear performance of Q\&P processing and low-temperature bainitic transformation for a medium-carbon dual-phase steel, Wear 402: 21-29.

Edmonds, D. V., He, K., Rizzo, F. C., De Cooman, B. C., Matlock, D. K., Speer, J. G. (2006). Quenching and partitioning martensite-A novel steel heat treatment. Materials Science and Engineering: A 438: 25-34.

Gao, G., Zhang, H., Tan, Z., Liu, W., Bai, B. (2013). A carbidefree bainite/martensite/austenite triplex steel with enhanced mechanical properties treated by a novel quenching- partitioning-tempering process. Materials Science and Engineering: A 559: 165-169.

HajyAkbary, F., Sietsma, J., Miyamoto, G., Furuhara, T., Santofimia, M. J. (2016). Interaction of carbon partitioning, carbide precipitation and bainite formation during the Q\&P process in a low $C$ steel. Acta Materialia 104: 72-83.

Hao, Q., Qin, S., Liu, Y., Zuo, X., Chen, N., Huang, W., Rong, $Y$. (2016). Effect of retained austenite on the dynamic tensile behavior of a novel quenching-partitioning-tempering martensitic steel. Materials Science and Engineering: A 662: 16-25.

Hauserová, D., Nový, Z., Dlouhý, J., Motyčka, P. (2011). Q-P processing of high-strength low-alloyed steel sheets. Metal'2011, Brno, Czech Republic, 1-5.

Karam-Abian, M., Zarei-Hanzaki, A., Abedi, H. R., HeshmatiManesh, S. (2016). Micro and macro-mechanical behavior of a transformation-induced plasticity steel developed by thermomechanical processing followed by quenching and partitioning. Materials Science and Engineering: A 651: 233-240.

Kolk, A. J. (2014). Is retained austenite controlling the mechanical properties of Q\&P steels?. Delft University of Technology, Faculty Mechanical, Department Materials Science and Engineering, Master Thesis $1 \mathrm{~s}$. Netherlands.

Krauss, G. (1999). Martensite in steel: strength and structure. Materials Science and Engineering: A 273: 40-57.

Liu, H., Jin, X., Dong, H., Shi, J. (2011). Martensitic microstructural transformations from the hot stamping, quenching and partitioning process. Materials characterization 62(2): 223-227.

Qu, H. (2013). Advanced high strength steel through paraequilibrium carbon partitioning and austenite stabilization. Doctoral Dissertation, Ph. D. Thesis, Case Western Reserve University, Cleveland, $\mathrm{OH}$.

Santofimia, M. J., Zhao, L., Sietsma, J. (2009). Microstructural evolution of a low-carbon steel during application of quenching and partitioning heat treatments after partial austenitization. Metallurgical and Materials Transactions A 40(1): 46.

Speer, J. G., De Moor, E., Clarke, A. J. (2015). Critical assessment 7: Quenching and partitioning. Materials Science and Technology 31(1): 3-9.

Tan, X., Xu, Y., Yang, X., Wu, D. (2014). Microstructure-properties relationship in a one-step quenched and partitioned steel. Materials Science and Engineering: A 589: 101-111.

Tariq, F., \& Baloch, R. A. (2014). One-step quenching and partitioning heat treatment of medium carbon low alloy steel. Journal of materials engineering and performance 23(5): 1726-1739.

Wang, C. Y., Shi, J., Cao, W. Q., Dong, H. (2010). Characterization of microstructure obtained by quenching and partitioning process in low alloy martensitic steel. Materials Science and Engineering: A 527(15): 3442-3449.

Wang, X. D., Xu, W. Z., Guo, Z. H., Wang, L., Rong, Y. H. (2010). Carbide characterization in a Nb-microalloyed advanced ultrahigh strength steel after quenching-partitioning-tempering process. Materials Science and Engineering: A 527(15): 3373-3378.

Wang, X. D., Guo, Z. H., Rong, Y. H. (2011). Mechanism exploration of an ultrahigh strength steel by quenching-partitioning-tempering process. Materials Science and Engineering: A 529: 35-40. 
Wang, L., Speer, J. G. (2013). Quenching and partitioning steel heat treatment. Metallography, Microstructure, and Analysis 2(4): 268-281.

Wang, C. Y., Chang, Y., Yang, J., Cao, W. Q., Dong, H. (2016). Work hardening behavior and stability of retained austenite for quenched and partitioned steels. Journal of Iron and Steel Research International 23(2): 130-137.

Yang, L. I., Lu, Y. P., Chong, W. A. N. G., Li, S. T., Chen, L. B. (2011). Phase stability of residual austenite in 60Si2Mn steels treated by quenching and partitioning. Journal of Iron and Steel Research, International 18(2): 70-74.

Zhou, S., Zhang, K., Wang, Y., Gu, J. F., Rong, Y. H. (2011). High strength-elongation product of $\mathrm{Nb}$-microalloyed lowcarbon steel by a novel quenching-partitioning-tempering process. Materials Science and Engineering: A 528(27): 8006-8012. 